\title{
Avaliação dos estoques pesqueiros de quatro espécies de grandes bagres (Siluriformes, Pimelodidae) na bacia do rio Cuiabá, Pantanal norte, Brasil, utilizando alguns Pontos de Referência Biológicos
}

\author{
Lúcia A. F. Mateus \& Jerry M. F. Penha
}

Laboratório de Ecologia e Manejo de Recursos Pesqueiros, Departamento de Botânica e Ecologia, Instituto de Biociências, Universidade Federal de Mato Grosso. 78060-900 Cuiabá, Mato Grosso, Brasil. E-mail: Imateus@ufmt.br

\begin{abstract}
Fisheries stock assessment of four large catfish (Siluriformes, Pimelodidae) in the Cuiaba River basin, north Pantanal, Brazil, using some Biological Reference Points. The growth and mortality parameters estimated from length structure were used to evaluate the state of explotation of the stocks of four large catfish species from Cuiabá River basin, north Pantanal. The species studied were the Flatwhiskered catfish, the Jaú, the Barred sorubim, and the Spotted sorubim. The assessment were conducted with the yield per recruit model, and the Biological Reference Points $F_{\max } F_{0.1}, F=M$, and $F_{B D R}$. The analysis indicates that the stocks of the Spotted sorubim, and of the Jaú are not overexploited. However, the stocks of the Barred sorubim, and, in lower degree, of the Flatwhiskered catfish of the Cuiabá River basin are in imminent threat of overfishing.

KEY WORDS. Beverton \& Holt model; fisheries management; overfishing; yield per recruit.
\end{abstract}

RESUMO. Os parâmetros de crescimento e mortalidade estimados a partir da estrutura em comprimento foram utilizados para avaliar o estado de explotação dos estoques de quatro espécies de grandes bagres da bacia do rio Cuiabá, Pantanal norte. As espécies estudadas foram o barbado, o cachara, o jaú e o pintado. As avaliações foram realizadas por meio do modelo de rendimento por recruta, considerando os Pontos de Referência Biológicos $\mathrm{F}_{\text {max }}{ }^{\prime}$ $\mathrm{F}_{0,{ }^{\prime}} \mathrm{F}=\mathrm{M} \mathrm{e}_{\mathrm{BDR}}$. As análises indicam que os estoques do pintado e do jaú estão bem conservados. Os estoques do cachara e, menos fortemente, o do barbado estão em iminente ameaça de sobrepesca na bacia do rio Cuiabá.

PALAVRAS-CHAVE. Manejo da pesca; modelo de Beverton \& Holt; rendimento por recruta; sobrepesca.

Avaliação de estoque pesqueiro é o nome dado a vários rituais usados para estimar o tamanho atual, a taxa de explotação e a produtividade potencial de um estoque (HILBORN 1992). O objetivo essencial desta avaliação é prover aconselhamento técnico para manter a produtividade do estoque de peixes e manejar a pesca para capturas, tão próximas quanto possível, da máxima teórica sustentável.

Pontos de Referência Biológicos (PRB), no contexto da avaliação de estoques, podem ser definidos como um valor convencional derivado de uma técnica de análise, o qual representa o estado do estoque ou população, e cujas características acredita-se ser útil para o manejo do estoque (CADDY \& MAHON 1995). Assim pontos de referência biológicos são níveis com os quais o atual estado do estoque pode ser medido (COLLIE \& GISLASON 2001). Em termos práticos são, freqüentemente, valores arbitrários e muitas vezes especificados sem variância.

Os PRB podem ser: (I) pontos de referência alvo, os quais indicam o estado desejado; ou (II) pontos de referência limite, os quais indicam a aproximação de um certo valor de perigo, o qual deve ser evitado.
A mortalidade por pesca (F) e a biomassa (B) do estoque são, geral mente, as variáveis de referência básicas utilizadas para se estimar os PRB, uma vez que, as relações entre $F$, B e rendimento fornecem a base para a maioria dos PRB (CADDY \& M AHON 1995). Um dos pontos de referência largamente aplicados tem sido a taxa de mortalidade que maximiza o rendimento sem, no entanto, levar ao risco de sobrepesca, ou seja, a taxa de mortalidade que permite o rendimento máximo sustentável (MSY) - referida como $F_{M S Y}$. Porém, para se estimar confiavelmente $F_{\text {MSY }}$ é preciso saber se há denso dependência no estoque, e qual a sua forma, que por sua vez pressupõe o conhecimento da relação estoque-recrutamento (CLARK 1991). Quando estas informações são desconhecidas a alternativa é utilizar como PRB as taxas de mortalidade por pesca (F) estimadas a partir dos parâmetros de história de vida.

Raramente um único ponto ésuficiente para o julgamento da condição de um estoque. Em adição, o mesmo ponto de referência pode ser categorizado diferentemente dependendo da espécie em questão e da percepção do investigador sobre a resiliência do estoque (MACE 1994). Assim, neste estudo, qua-

Revista Brasileira de Zoologia 24 (1): 144-150, março 2007 
tro Pontos de Referência Biológicos foram utilizados para avaliação dos estoques de quatro espécies de grandes bagres da bacia do rio Cuiabá, Pantanal norte, a saber: o barbado, Pinirampus pirinampu Spix \& Agassiz (1829), o cachara, Pseudoplatystoma fasciatum Linnaeus (1766), o jaú, Zungaro jahu Ihering (1898) e o pintado, Pseudoplatystoma corruscans Spix \& Agassiz (1829). Os dados são discutidos em comparação com o a taxa de mortalidade por pesca atual $\left(\mathrm{F}_{\text {atual }}\right)$.

\section{MATERIAL E MÉTODOS}

O Pantanal Mato-grossense é uma planície alagável com aproximadamente $140000 \mathrm{~km}^{2}$ e está sujeita a alternância de períodos de seca e cheia. Pertence a bacia hidrográfica do Alto rio Paraguai que nasce na Serra dos Parecis (região norte de Mato Grosso) e segue em direção sul com certa sinuosidade até Porto Murtinho (Mato Grosso do Sul).

$\mathrm{O}$ rio Paraguai recebe águas de vários afluentes que o alcançam com pouca velocidade e muitos sedimentos, que com as inundações vão se depositar na planície pantaneira. Os principais tributários em território brasileiro são: à direita - rios Jauru, Cabaçal e Sepotuba; à esquerda - rios Cuiabá, Taquari, Miranda e Apa.

Os dados de desembarque pesqueiro (em $\mathrm{kg}$ ) mensal e anual, por espécie, foram obtidos no Mercado do Peixe em Cuiabá, Mato Grosso, registrados pela Policia Ambiental do Estado de Mato Grosso. Os dados de desembarque foram obtidos para o período entre agosto e outubro de 2000 e entre março e outubro de 2001, juntamente com amostras mensais de comprimento furcal $(\mathrm{Lf}, \mathrm{cm})$ para as quatro espécies. Para os meses de novembro a fevereiro não existem informações devido ao período de defeso de reprodução. Todos os exemplares amostrados eram provenientes da pesca profissional-artesanal realizada na bacia do rio Cuiabá, Pantanal norte.

Para a análise de diferenças relativas de rendimento por recruta (Y/R) para diferentes valores de mortalidade por pesca $(F)$, utilizou-se o modelo relativo de rendimento por recruta (Y/R') (BEVERTON \& HOLT 1966). Este modelo permite a estimativa do rendimento potencial de um estoque em função da mortalidade por pesca (F) e do comprimento de primeira captura (Lci). Também permite que se avalie a resposta do rendimento por recruta a variações em F e Lci (Sparre \& Venema 1997).

$O$ rendimento relativo por recruta $\left(Y / R^{\prime}\right)$ e a biomassa relativa por recruta $\left(B / R^{\prime}\right)$ podem ser estimados pelas seguintes expressões: $Y / R^{\prime}=E^{m}\left[1-(3 U / 1+m)+\left(3 U^{2} / 1+2 m\right)-\left(U^{3} / 1+3 m\right)\right]$ e $\mathrm{B} / \mathrm{R}^{\prime}=(1-\mathrm{E})(\mathrm{a} / \mathrm{b}) ;$ onde: $\mathrm{U}=1-(\mathrm{Lci} / \mathrm{L} \infty) ; \mathrm{m}=(1-\mathrm{E}) /(\mathrm{M} / \mathrm{k})=\mathrm{k} / \mathrm{Z}$; $E=F / Z ; a=\left[1-(3 U / 1+m)+\left(3 U^{2} / 1+2 m\right)-\left(U^{3} / 1+3 m\right)\right] ; b=[1-(3 U /$ $\left.\left.1+m^{\prime}\right)+\left(3 U^{2} / 1+2 m^{\prime}\right)-\left(U^{3} / 1+3 m^{\prime}\right)\right] ; m^{\prime}=1 /(M / k)=m /(1-E)$, onde: Lci é o comprimento do peixe na primeira captura; $L \infty$ e k são parâmetros da equação de Von Bertalanffy e M é a mortalidade natural; F é a mortal idade por pesca; $Z$ é a mortalidade total e $\mathrm{E}$ é a taxa de explotação.

Considerando que $E=F / Z$, pode-se estimar $F$ a partir de $E$ pela seguinte expressão: $F=(M * E) /(1-E)$.

Devido à suposição de $M$ constante, o modelo foi simulado para diferentes valores de $\mathrm{M}$ para cada espécie.

Os parâmetros de crescimento ( $L \infty$ e k) e mortalidade (M eZ) utilizados aqui foram estimados por Mateus \& Penha (2007).

Os Pontos de Referência Biológicos (PRB) utilizados neste estudo para a avaliação dos estoques, em comparação com a taxa de mortalidade por pesca atual $\left(F_{\text {atual }}\right)$, foram: 1$) F_{\text {max }}$ - taxa de mortalidade por pesca que maximiza o rendimento por recruta sem considerar se o estoque desovante é conservado para manter o recrutamento no futuro. Estimado a partir de $\mathrm{E}_{\max } ; 2$ ) $\mathrm{F}_{0,1}$ - taxa na qual a inclinação da curva de rendimento por recruta cai para $10 \%$ de seu valor na origem (GulLand \& Boerema 1973). Estimado a partir de $E_{0,1}$; 3) $F=M$ - baseado na suposição de que a $\mathrm{F}_{\text {MSY }}$ é muitas vezes aproximadamente igual à mortalidade natural e; 4) $\mathrm{F}_{\mathrm{BDR}}$ - taxas de mortalidade por pesca referentes à análise da biomassa desovante por recruta, ou seja, $\mathrm{F}$ a qual reduz a biomassa inicial (sem explotação onde $F=0$ ) do estoque a um determinado nível $\left(\mathrm{F}_{35 \%}\right.$ e $\mathrm{F}_{40 \%}$ neste caso).

\section{RESULTADOS}

Com base nas curvas de rendimento relativo por recruta, e considerando os valores atuais de F, Lci e M (Tab. I), observase que os estoques do cachara e barbado estão sob ameaça de sobrepesca, pois já estão muito próximos do rendimento máximo (Fig. 1). Por outro lado, tanto o jaú, quanto o pintado, estão numa situação menos ameaçadora.

Considerando a possibilidade de aumento na mortalidade por pesca, uma projeção pode ser feita para o aumento do rendimento relativo por recruta em relação ao aumento em $\mathrm{F}$. Tal análise mostra que, para todas as espécies estudadas, um aumento em $\mathrm{F}$ não é acompanhando por um aumento igual no

Tabela I. Estimativas utilizadas para a aplicação do modelo de Beverton \& Holt e os resultados obtidos para os estoques do barbado, cachara, jaú e pintado na bacia do rio Cuiabá, Mato Grosso.

\begin{tabular}{|c|c|c|c|c|c|c|c|c|c|c|c|}
\hline & \multicolumn{5}{|c|}{ Entrada } & \multicolumn{6}{|c|}{ Saída } \\
\hline & Lci (cm) & $\mathrm{M}\left(\mathrm{ano}^{-1}\right)$ & $\mathrm{k}\left(\mathrm{ano}^{-1}\right)$ & $\mathrm{L}(\mathrm{(cm})$ & $\mathrm{Z}\left(\mathrm{ano}^{-1}\right)$ & $\mathrm{E}_{\max }$ & $\mathrm{E}_{0,1}$ & $E_{\text {atual }}$ & $\mathrm{F}_{\max }\left(\mathrm{ano}^{-1}\right)$ & $\mathrm{F}_{0,1}\left(\mathrm{ano}^{-1}\right)$ & $\mathrm{F}_{\text {atual }}\left(\mathrm{ano}^{-1}\right)$ \\
\hline Barbado & 42 & 0,58 & 0,300 & 90,6 & 1,155 & 0,739 & 0,659 & 0,497 & 1,64 & 1,12 & 0,574 \\
\hline Cachara & 60 & 0,31 & 0,131 & 122,4 & 0,657 & 0,842 & 0,767 & 0,528 & 1,65 & 1,02 & 0,347 \\
\hline Jaú & 82 & 0,28 & 0,128 & 172,0 & 0,465 & 0,803 & 0,707 & 0,398 & 1,14 & 0,67 & 0,185 \\
\hline Pintado & 57 & 0,29 & 0,127 & 149,7 & 0,427 & 0,642 & 0,553 & 0,321 & 0,52 & 0,36 & 0,137 \\
\hline
\end{tabular}



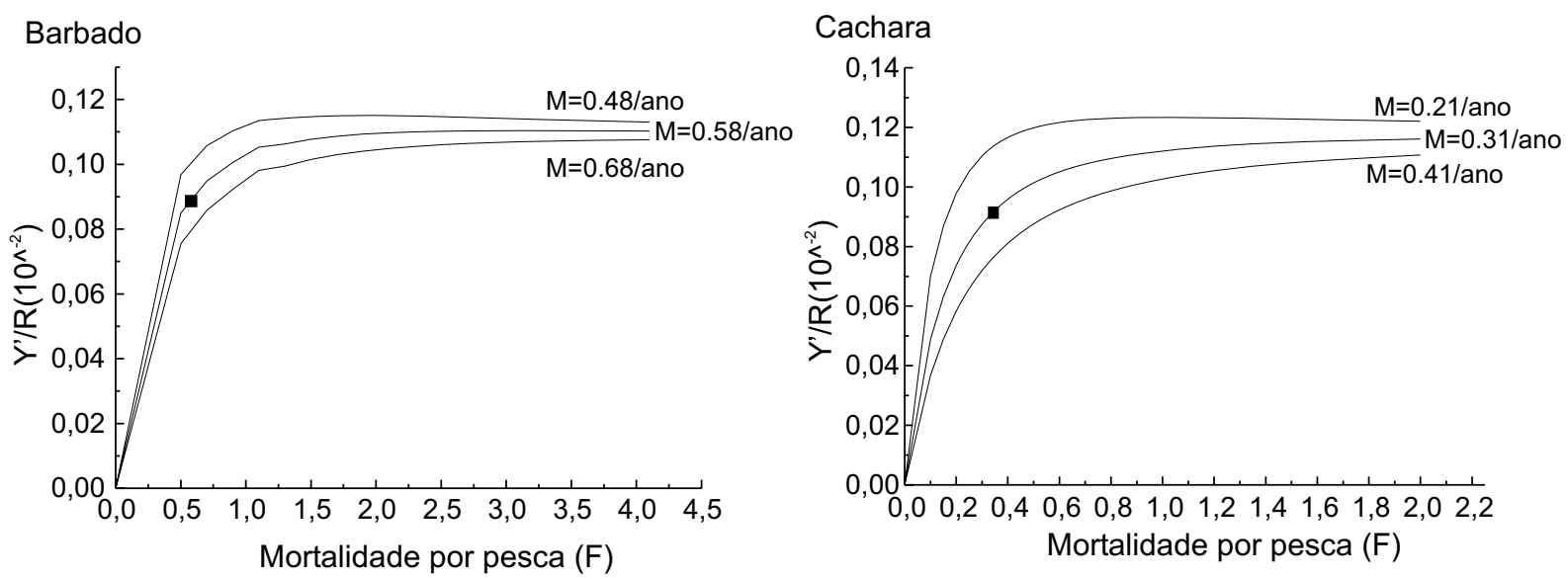

Jaú
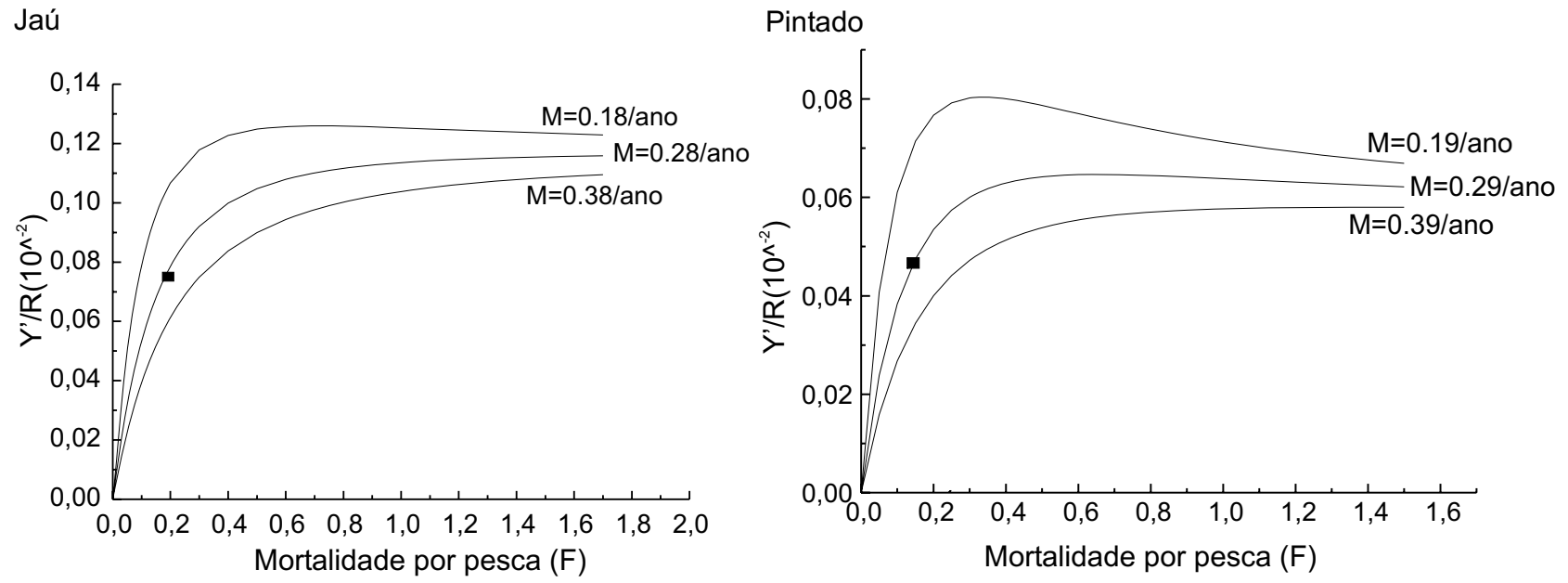

Figura 1. Curva de rendimento relativo por recruta para valores diferentes de $\mathrm{F}$ e $\mathrm{M}$ do barbado, cachara, jaú e pintado na bacia do rio Cuiabá, Mato Grosso. ( $\mathbf{\square}$ indica o estado atual do estoque).

rendimento. Ao contrário, quanto maior é o aumento em $\mathrm{F}$, menor é o incremento no rendimento (Fig. 2). Desse modo um aumento de $100 \%$ em $\mathrm{F}$ induz a um aumento menor que $20 \%$ no rendimento relativo por recruta para o barbado e o cachara, e em torno de $20 \%$ no caso dos estoques do jaú e pintado.

Neste trabalho, somente $F_{\max }$ é considerado um PRB limite, sendo $F_{0,1}, F=M$ e $F_{B D R}\left(F_{35 \%}\right.$ e $\left.F_{40 \%}\right)$ classificados como PRB alvo. Os resultados mostram que $F_{\max }$ não foi atingido por nenhuma das espécies anal isadas (Tab. II), assim como $F_{0,1}$, o qual foi relativamente maior que os outros PBR alvos. No entanto, os $\mathrm{F}_{\text {atual }}$ estimados para o barbado e o cachara são maiores que seus respectivos $\mathrm{F}_{\mathrm{BDR}}$. Para o barbado $\mathrm{F}_{\text {atual }}$ é aproximadamente igual à estimativa de sua mortalidade natural e para o cachara $F_{\text {atual }}$ é maior do que sua $M$ estimada. Por outro lado $F_{\text {atual }}$ obtida para o jaú e o pintado está abaixo de todos os PRBs utilizados.

Assim, adotando-se uma posição conservativa pode-se concluir que o estoque do cachara está em iminente ameaça de sobrepesca, uma vez que seu $F_{\text {atual }}$ já ultrapassou dois dos três

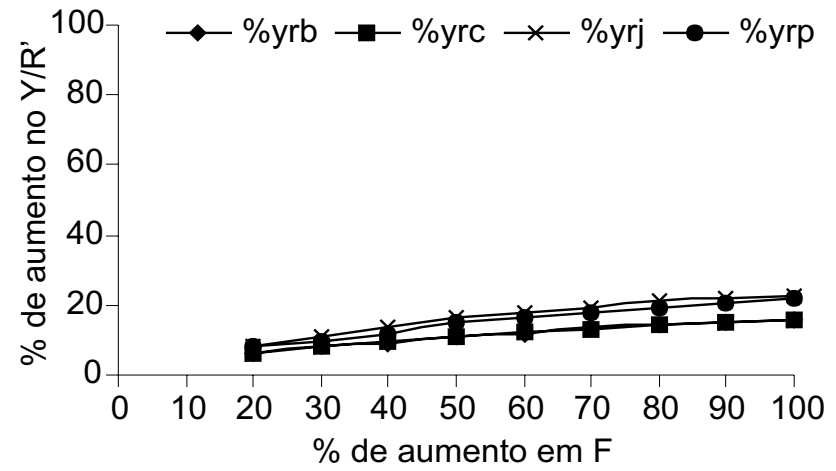

Figura 2. Relação entre a porcentagem de aumento na mortalidade por pesca e o aumento do rendimento pesqueiro, considerando as estimativas pelo modelo de Beverton \& Holt (B) para os estoques do barbado, cachara, jaú e pintado na bacia do rio Cuiabá, Mato Grosso. (Yb e yrb) Barbado, (Yc e yrc) cachara, (Yj e yrj) jaú, (Yp e yrp) pintado. 
Tabela II. Taxa de mortalidade por pesca atual e pontos de referência biológicos (PRB) baseados em $\mathrm{F}$ utilizados na avaliação dos estoques do barbado, cachara, jaú e pintado na bacia do rio Cuiabá, Mato Grosso.

\begin{tabular}{lcccccc}
\hline & $\begin{array}{c}\text { Fatual } \\
\left(\mathrm{ano}^{-1}\right)\end{array}$ & $\begin{array}{c}\mathrm{Fmax} \\
\left(\mathrm{ano}^{-1}\right)\end{array}$ & $\begin{array}{c}\mathrm{F} 0,1 \\
\left(\mathrm{ano}^{-1}\right)\end{array}$ & $\begin{array}{c}\mathrm{F}=\mathrm{M} \\
\left(\mathrm{ano}^{-1}\right)\end{array}$ & $\begin{array}{c}\mathrm{F} 35 \% \\
\left(\mathrm{ano}^{-1}\right)\end{array}$ & $\begin{array}{c}\mathrm{F} 40 \% \\
\left(\mathrm{ano}^{-1}\right)\end{array}$ \\
\hline Barbado & 0,574 & 1,64 & 1,12 & 0,58 & 0,553 & 0,457 \\
Cachara & 0,347 & 1,65 & 1,02 & 0,31 & 0,316 & 0,264 \\
Jaú & 0,185 & 1,14 & 0,67 & 0,28 & 0,282 & 0,234 \\
Pintado & 0,137 & 0,52 & 0,36 & 0,29 & 0,240 & 0,204 \\
\hline
\end{tabular}

PRBs alvos estimados. Da mesma forma, mas com menos evidência, pois seu $F_{\text {atual }}$ ultrapassou somente um dos três PRBs alvos estimados, o estoque do barbado também está sob iminente ameaça de sobrepesca. Em relação aos estoques do jaú e do pintado a situação é menos preocupante, pois $F_{\text {atual }}$ nos dois casos está aquém dos PRBs alvos (Fig. 3).

\section{DISCUSSÃO}

A base do modelo de rendimento por recruta (Y/R) é considerar o efeito de diferentes estratégias de explotação, assumindo o estado de equilíbrio, relacionando a captura de um Iado e crescimento e mortalidade de outro, tentando estimar a mortalidade por pesca $\left(\mathrm{F}_{\max }\right)$ onde o rendimento máximo por recruta que garantiria a reposição do estoque (MSY) seria alcançado. Assim não extrapolando esse nível de explotação o estoque, teoricamente, não declinaria (Beverton \& Holt 1966).

O conceito de $\mathrm{Y} / \mathrm{R}$ pode ser usado satisfatoriamente somente em conjunto com informações adicionais ou suposições sobre o nível apropriado de mortalidade por pesca ou biomassa do estoque. Estes níveis são pontos de referência com os quais o status atual do estoque pode ser avaliado. Assim, pontos de referência biológicos (PRB) são amplamente utilizados para definir níveis seguros de pesca para populações de peixes (ColLIE $\&$ GISLASON 2001).

Neste estudo os PRBs utilizados para a avaliação dos estoques do barbado, cachara, jaú e pintado na bacia do rio Cuiabá, são baseados em estimativas de níveis de mortalidade por pesca: $F_{\text {max }}, F_{0,1}, F=M$ e $F_{B D R}$. Quando comparados com o $F_{\text {atual }}$, os PRBs fornecem indicações sobre a situação do estoque frente ao regime de pesca praticado. Esta avaliação é essencial para o estabelecimento de planos de manejo para as espécies em questão.

Tais PRBs foram escolhidos em função do tipo de dados disponível acerca da pesca destas espécies, ou seja, dados de rendimento e biomassa por recruta estimados a partir de parâmetros de crescimento, tamanho de primeira captura e mortalidade. A análise de Y/R somente se aplica a longo prazo, ou em situação de equilíbrio. Entretanto, infelizmente, devido a ausência de dados de CPUE e informações sobre a relação estoque recrutamento, outros pontos de referência não puderam ser estimados.

As quatro espécies aqui avaliadas não apresentaram valo- res de suas respectivas mortalidades que maximizem o rendimento por recruta $\left(F_{\max }\right)$. $F_{\max }$ foi considerada como um PRB limite, pois, utilizá-la como PRB alvo é indesejável por várias razões, dentre elas o fato da curva de rendimento por recruta tender a um platô nas vizinhanças do ponto máximo (DERIso 1987). Assim, atingir $F_{\text {max }}$, a qual é relativamente alta (como foi encontrado para as espécies avaliadas), requereria um grande aumento no esforço de pesca levando a ineficiência econômica.

Ademais, quando existe uma relação clara entre estoque e recrutamento, $F_{\max }$ é maior que a taxa de mortalidade por pesca que produz o MSY, levando ao risco de sobrepesca de recrutamento se esta for atingida. Além disso, como $F_{\max }$ é tipicamente alta, existe pouca dúvida de que ela não exceda $F_{\text {MSY }}$ (CLARK 1991). Assim deve-se garantir através de planos de manejo que este PRB não seja alcançado por nenhum dos estoques aqui avaliados, como já vem ocorrendo com o pacu, Piaractus mesopotamicus, na porção sul do Pantanal (CATTELA et. al. 2005).

Em estoques onde a relação estoque/recrutamento é desconhecida, um limite arbitrário de $\mathrm{F}$ tem sido proposto: $\mathrm{F}_{0.1}$. $\mathrm{O}$ conceito de $F_{0,1}$ foi introduzido por GuLLAND \& Boerema (1973), baseado em considerações econômicas, como um arbitrário, mas consistente, ponto de referência abaixo do rendimento máximo. Tal taxa de mortalidade por pesca é o valor de $\mathrm{F}$ que corresponde a $1 / 10$ da taxa de incremento, ou seja, um limite arbitrário de $\mathrm{F}$ em um nível tal que o incremento no Y/R é $10 \%$ de seu valor próximo à origem da curva (DERISO 1987). Considerado um PRB alvo (CAdDy \& Mahon 1995, Collie \& Gislason 2001), $F_{0,1}$ invariavelmente é menor que $F_{\max }$ e, usualmente, fornece um valor próximo ao $\mathrm{F}_{\mathrm{MSY}}$. Assim, é menos provável que dirija o estoque a depleção (CLARK 1991).

As análises conduzidas neste estudo indicaram que os estoques do barbado, cachara, jaú e pintado não alcançaram o valor de $\mathrm{F}_{0,1}$ estimado, considerando a taxa de mortalidade natural estimada pela equação de Pauly. Entretanto deve-se ter em mente que $F_{0,1}$ muda com o padrão de pesca e com o valor de entrada de M. Assim não têlo atingido ainda, não significa necessariamente que o esforço de pesca possa ser aumentado para todas as espécies em questão, sem prejuízo para a renovação de seus estoques. Além disto, é preciso levar em consideração, por exemplo, qual a porcentagem de redução que este nível de pesca traria para a biomassa por recruta inicial (quando $F=0$ ). Dentro desta perspectiva o limite ótimo estaria entre 20 e $30 \%$ da biomassa do estoque virgem (Quinn II et al. 1990) e em estoques pouco conhecidos, principalmente em relação ao estoque-recrutamento como neste caso, este limite deveria ser 30\% (CADDY \& MAHON 1995). Esta recomendação é sustentada pela análise teórica de MACE (1994). Para os estoques do barbado, cachara, jaú e pintado a redução em $B / R$ aplicando-se $F_{0,1}$ seria para menos de $30 \%$ do estoque virgem $(20 \%, 12,75 \%, 17 \%$ e $24,7 \%$, respectivamente), e dessa forma ultrapassaria o limite recomendado podendo colocar em risco os estoques. Assim, até que se obtenha informações sobre estoque-recrutamento, não é aconselhável aumentar o esforço de pesca em direção à $F_{0,1}$.

Revista Brasileira de Zoologia 24 (1): 144-150, março 2007 


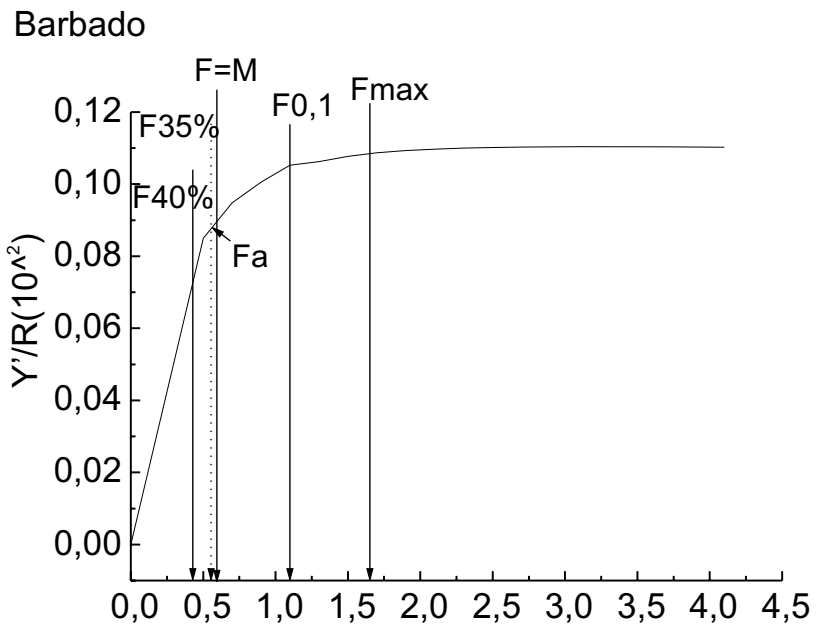

Jaú

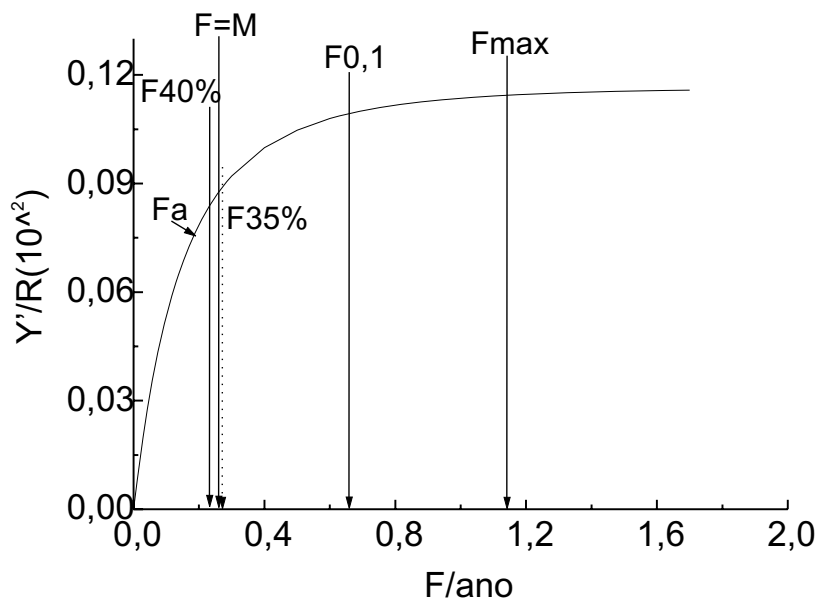

\section{Cachara}

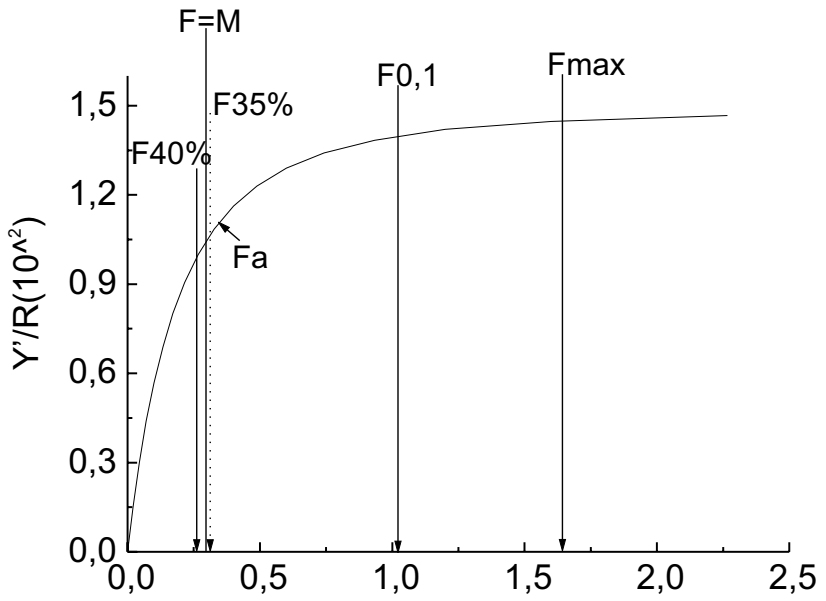

Pintado

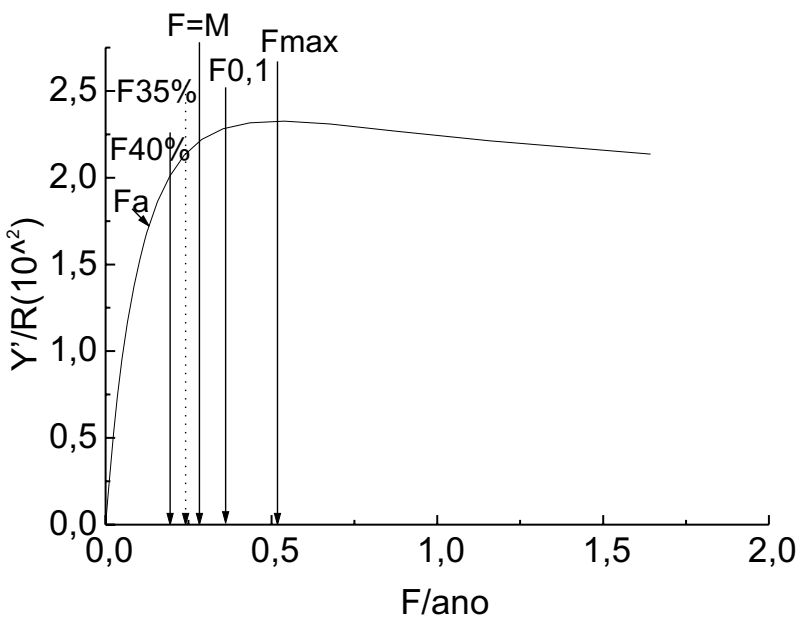

Figura 3. Relação entre o rendimento relativo por recruta, a taxa de mortalidade por pesca atual (Fa) e os pontos de referência biológi$\cos$ (PRB) baseados em F utilizados na avaliação dos estoques do barbado, cachara, jaú e pintado na bacia do rio Cuiabá, Mato Grosso.

Tem-se assumido que no MSY, F é igual a M. Na forma logística essa relação é verdadeira se o recrutamento no MSY é igual ao que ocorre no estoque não explorado em equilíbrio. Isto tenderia a indicar recrutamento relativamente constante ou independente da densidade (FrancIs 1974). Porém, existem poucas evidências empíricas de tal relação entre $M$ e F, de modo que o $F_{\text {MSY }}$ poderia ser maior que $M$ em alguns casos (CLARK 1991, CADDY \& MAHon 1995). Por outro lado, o rendimento total pode ser maximizado pela pesca em nível moderado, sendo importante reconhecer que grandes estoques, altos rendimentos, melhor estabilidade e alto lucro, somente podem ser mantidos se o esforço é mantido baixo indefinidamente (SHEPHERD 1990). Desse modo, ao considerar a relação de igualdade entre $\mathrm{M}$ e F como um PRB alvo para este estudo, pode-se sugerir que as pescarias do barbado e do cachara já atingi ram este objetivo, ou seja, seus estoques estão sendo explorados no nível máximo sustentável (sendo que para o cachara $F_{\text {atual }}$ é ligeiramente maior que M ). Já em relação aos estoques do jaú e do pintado a taxa de mortalidade por pesca poderia aumentar se houvesse viabilidade econômica.

Outro PRB alvo aplicado neste trabal ho é a taxa de mortalidade por pesca referente à análise da biomassa por recruta, ou seja, $F$ que reduz a biomassa por recruta do estoque virgem a uma determinada porcentagem $\left(\mathrm{F}_{\mathrm{BDR}}\right)$ que permite a explotação do estoque próximo ao MSY para qualquer provável relação estoque-recrutamento. Este nível sustentável de pesca estaria em torno do $\mathrm{F}_{35 \%}$, que reduz a biomassa por recruta a $35 \%$ de seu nível não explorado para peixes de fundo (CLARK 1991). Entretanto, quando a relação estoque-recrutamento é desconhecida, $\mathrm{F}_{40 \%}$ deveria ser adotada como taxa de mortalidade por pesca 
alvo (MACE 1994). As atuais taxas de mortalidade por pesca do barbado e do cachara encontram-se, ligeiramente, acima destes dois níveis de $\mathrm{F}_{\mathrm{BDR}}$, portanto em iminente ameaça de sobrepesca. Para os estoques do jaú e do pintado os valores estimados de $F_{\text {atual }}$ são um pouco menores que $F_{35 \%}$ e $F_{40 \%}$, portanto não tendo ainda atingido seu MSY. Em síntese, os dados revelam que na bacia do rio Cuiabá os estoques do barbado e do cachara encontram-se em risco iminente de sobrepesca, enquanto que os estoques do jaú e do pintado ainda não estão sob esta ameaça.

Estudos de avaliação de estoques pesqueiros real izados na porção sul do Pantanal, com base em dados obtidos entre 1994 e 1999, não encontraram evidências de sobrepesca para o barbado, cachara e pintado (CATELLA et. al. 2005). No entanto, em tal estudo o conjunto de dados não permitiu um resultado conclusivo para o estoque do jaú, sugerindo que talvez este esteja na iminência de sobrexplotação. Porém, é preciso levar em conta que o modelo de análise utilizado no estudo de CATELLA et al. (2005) - o modelo de produção excedente, considera a relação entre a captura e o esforço de pesca, assumindo que a captura é proporcional a biomassa do estoque. Estes modelos necessitam de uma longa série temporal de dados e a sobrexplotação só é detectada após o MSY ter sido ultrapassado (HILBORN \& W ALTERS 1992). Talvez, devido ao fato dos estoques do barbado e cachara estarem sendo explotados em níveis muito próximos ao MSY, a sua iminente ameaça não tenha sido detectada pelo modelo de produção excedente. Há ainda a possibilidade de se tratar de estoques diferentes. Porém, em se tratando de espécies migradoras, está última hipótese seria pouco provável.

Em suma, estas inferências levantadas só começarão a ser esclarecidas a partir de estudos mais aprofundados sobre a estrutura dos estoques. Ou seja, quantos estoques de cada uma das espécies estudadas existem no Pantanal? E ainda, estudos da relação estoque-recrutamento, que permitirão uma estimativa mais acurada da capacidade de renovação dos estoques em conjunto com o aprofundamento no conhecimento acerca da história de vida das espécies.

Com base nas análises acima e adotando uma posição mais conservativa, uma vez que, dados de CPUE e relação estoque-recrutamento inexistem para as espécies aqui estudadas, quais seriam as recomendações mais apropriadas acerca do manejo destas espécies na bacia do rio Cuiabá?

A resposta para esta questão não é simples, mas nestes termos, adotando-se uma posição conservadora, as taxas de mortalidade por pesca sobre os estoques do barbado e do cachara deveriam ser reduzidas, aproximadamente, em $20 \%$ para o primeiro e $25 \%$ para o segundo, a fim de evitar qualquer ameaça de sobrepesca diante dos riscos e incertezas que cercam a avaliação dos estoques. Em relação aos estoques do jaú e do pintado, apesar dos resultados apontarem para a possibilidade de um aumento em $\mathrm{F}$ em torno de $20 \%$ e $30 \%$, respectivamente, o acréscimo que este aumento proporcionaria ao rendimento por recruta seria inferior a $10 \%$. Portanto, considerando que um aumento em F implicaria em um aumento igual no esforço de pesca, do ponto de vista econômico, provavelmente, não seria atrativo aumentar F para estas espécies, podendo então ser mantido nos níveis atuais.

Entretanto a decisão acerca da viabilidade destas recomendações e quais estratégias seriam mais adequadas para assegurá-las, depende de uma ampla discussão conjunta entre todos os interessados no recurso, como por exemplo, pescadores, pesquisadores e governo no papel de manejadores. Primeiro para o estabelecimento dos objetivos do manejo da pesca e posteriormente dos meios político-institucionais para se alcançar tais objetivos, o que sempre é uma tarefa complexa. Ainda é preciso levar em consideração que a sobrepesca pode ser evitada através de medidas que garantam que a explotação mantenha-se em níveis populacionalmente aceitáveis, permitindo a renovação dos estoques. No entanto a renovação dos estoques é fortemente dependente da qualidade ambiental. Dessa forma, primeiramente é preciso considerar os fatores que determinam a qualidade do ambiente do ponto de vista das populações de peixes. Assim em áreas alagáveis podemos elencar três pontos fundamentais: 1) complexidade do habitat; 2) qualidade da água; 3) o pulso de inundação. Portanto qualquer atividade que afete estes fatores implicará em al terações na produção de peixes e, portanto, na atividade pesqueira. Assim para que a pesca seja sustentável o ambiente deve ser conservado.

\section{AGRADECIMENTOS}

Aos pescadores do rio Cuiabá e aos comerciantes de peixes do mercado "Antônio Moysés Nadaf"; à Fundação de Amparo à Pesquisa do Estado de Mato Grosso - FAPEMAT, ao Centro de Pesquisas do Pantanal - CPP/MCT; à UFMT e à CAPES. Este estudo é parte da tese de doutorado de L. Mateus, realizada junto ao Programa dePós Graduação em Zoologia, da UNESP, campus de Rio Claro, sob a orientação de Miguel Petrere Jr.

\section{REFERÊNCIAS BIBLIOGRÁFICAS}

Beverton, R.J.H. \& S.J. Holt. 1966. Manual of methods for fish stock assessment. Part 2: tables of yield functions. FAO Fisheries Technical Paper 38: 1-67.

CADDY, J.F. \& R. MAHON. 1995. Reference points for fisheries management. FAO Fisheries Technical Paper 347: 1-83.

Catella, A.C.; F.F. Albuquerque \& F.L.R. Campos. 2005. sistema de controle da pesca de Mato Grosso do Sul SCPESCA/ MS - 6 - 1999. Corumbá, Embrapa Pantanal, 60p.

CLARK, W.G. 1991. Groundfish exploitation rates based on life history parameters. Canadian Journal of Fisheries and Aquatic Science 48: 743-750.

Collie, J. S. \& H. Gislason. 2001. Biological reference points for fish stocks in a multispecies context. Canadian Journal of Fisheries and Aquatic Science 58: 2167-2176.

Deriso, R.B. 1987. Optimal $F_{0,1}$ criteria and their relationship to maximum sustainable yield. Canadian Journal of Fisheries and Aquatic Science 44: 339-348.

Revista Brasileira de Zoologia 24 (1): 144-150, março 2007 
FrANCIS, R.C. 1974. Relationship of fishing mortality to natural mortality at the level of maximum sustainable yield under the logistic stock production model. Journal of Fishery Research Board of Canada 31: 1539-1542.

Gulland, J.A \& L.K. Boerema 1973. Scientific advice on catch levels. Fishery Bulletin 71: 325-335.

HilboRn, R. 1992. Current and future trends in fisheries stock assessment and management. South African Journal of Marine Science 12: 975-988.

Hilborn, R. \& C.J. W Alters. 1992. Quantitative fisheries stock assessment: choice, dynamics and uncertainty. New York, Chapman and Hall, 570p.

MACE, P.M. 1994. Relationships between common biological reference points used thresholds and targets of fisheries management strategies. Canadian Journal of Fisheries and
Aquatic Science 51: 110-122.

Mateus, L.A.F. \& J.M.F. Penha. 2007. Dinâmica populacional de quatro espécies de grandes bagres na bacia do rio Cuiabá, Pantanal norte, Brasil (Siluriformes, Pimelodidae). Revista Brasileira de Zoologia 24 (1): 87-98.

QUinn II, T.J.; R. FAJEN \& J. ZHENG. 1990. Threshold management policies for exploited populations. Canadian Journal of Fisheries and Aquatic Science 47: 2016-2029.

SHePHERD, J.G. 1990. Stability and the objectives of fisheries management: the scientific background. Lowestoft, Laboratory Leaflet, MAFF Directory of Fisheries Research, vol. 64, 16p.

SparrRe, P. \& S.C. Venema. 1997. Introdução à avaliação de mananciais de peixes tropicais. Parte 1: manual. Roma, FAO, 404p.

Recebido em 21.VI.2006; aceito em 28.II.2007. 